\title{
A MULTIDISCIPLINARY DOCUMENTAL REPRESENTATION METHOD FOR KINETIC AND ENVIRONMENTAL ART
}

\author{
F. Gasparetto ${ }^{*}$, A. Devecchi², L. Baratin ${ }^{2}$ \\ ${ }^{1}$ DICEA, via Brecce Bianche, University Politecnica delle Marche, Ancona AN, fnc.gasparetto@gmail.com \\ ${ }^{2}$ Conservation and Restoration school, DiSPeA, Piazza della Repubblica, 13, University of Urbino PU, alice.devecchi@gmail.com, \\ laura.baratin@uniurb.it
}

Commission II, WG II/8

KEY WORDS: digital documentation, environmental artwork, contemporary art, survey, representation

\begin{abstract}
:
The contribution addresses the definition of a new collaborative documental method for designing and managing the different phases of conservation of kinetic-programmed art. Our approach consists of developing a new representative model that includes both mechanical parts and spatial characteristics. The research stems from a specific case-study, Ambiente - Strutturazione a parametri virtuali (1969) by Gabriele Devecchi, permanently displayed at Museo del '900 in Milan since 2010. Starting from the dimensional and technical data, we obtained a graphical model of lamps 2D and 3D. They were enriched by a detailed abacus describing all the elements and by specific maps capturing all the phases of regular and extraordinary maintenance underwent by the environment.

The second part was carried out with a report about physical motion. The goal has been representing speed and geometry of the movement inside the space. By merging the first part of the documenting process and the second one we've got a graphic digital model including information about the individual parts of the installation and their mechanical interaction. The third and last step is ongoing and tackles the challenge of using virtual technologies for the description of the whole environment. Thanks to a collaboration between technicians and theoretic scholars, we attempted to match the study of the physical motion and all data about the structural parts with the careful consideration of historical-artistic and perception-related features. The work led to the conclusion that a virtual, immersive reproduction of the environment is not enough for deeply understanding the experience enjoyed by users inside it, because it misses the embodied perception activated by the artwork. For this reason, this study may be considered as a step in a broader research path about documentation of complex environmental, immersive, kinetic works of art.
\end{abstract}

\section{INTRODUCTION}

The issue of documentation is a growing concern in the contemporary art discourse. Since art started to focus on concepts and ideas more than on materials and techniques, conservation professionals have questioned the existing methods of artworks' documentation in search of strategies for managing the complex and often puzzling nature of contemporary art. The present contribution is part of an ongoing effort of defining and experimenting good practices of documentation for a specific typology of artwork, i.e. a kineticprogrammed environment. The reasons why taking the challenge include on one hand the need of conserving an artwork which, in order to preserve its identity, must be kept in function and, precisely because of the movement, is subject to fast deterioration. On the other hand, the need of re-installing the artwork for temporary exhibitions urges us to develop a unique methodology for a proper reconstruction on each occasion. In this perspective, we focused particularly on the output of the documentation process addressing an effective representation of material and immaterial aspects of the artwork. To this aim, many different professionals have been called upon for their specific expertise, so to elaborate a documentation useful both for conservation and for the future fruition of the artwork.

The case study analyzed in the essay - Ambiente Strutturazione a parametri virtuali by Gabriele Devecchi (Milan 1938 - 2011), 1969 - is included in a broader research about conservation and restoration of moving artworks by the same artist, led within the
School of Conservation and Restoration of the Cultural Heritage, Università di Urbino "Carlo Bo" together with Archivio Gabriele Devecchi. Within this frame, in the past years two other pieces by Devecchi - i.e. Superficie in vibrazione (1969) and Cubo reticolo in variazione (1965-1966) - have been investigated. In both cases the main challenges have been the recovery of the original functioning and planning of conservation that could ensure their fruition in movement. Nevertheless, both the artworks consisted in small objects. Otherwise, the case study analyzed in this essay has an environmental scale. As an environment, it raises additional questions connected to the spatial dimension, the dynamic interaction with the user and a general higher complexity of its parts.

For these reasons also, documentation-related actions have been carried out with the contribution of different expertise, crucial for handling a large amount of data of different nature, their storage and representation. In fact, the resulting documentation endeavours to hold together many features of equal importance as geometrical proportions, mechanical aspects and, of course, fruition-related issues that represent the tougher challenge.

The research succeeded in developing a multidisciplinary documentative booklet that could assist the decision making process in case of maintenance - be it ordinary or extraordinary - conservative measures, interventions of restoration, but also eventual re-installations of the above mentioned environmental artwork.

\footnotetext{
* Corresponding author
} 


\section{DIGITAL DOCUMENTATION FOR THE CONSERVATION OF CONTEMPORARY ARTWORKS}

\subsection{State of art}

Documentation of contemporary art is growing as a broad disciplinary field as long as art is increasing its complexity in terms of both media adopted and contents expressed. The inclusion of everyday materials in the artworks, actually dating back to the early XX Century and evolving since then towards more and more ephemeral media, pushed conservation science to investigate and test suitable methods for preserving the intangible features of the artworks, alongside the tangible ones.

According to Gunnar Heydenreich (2011) «the experience of the transience of modern materials and media has promoted an awareness of the importance of documentation». In fact, documentation is accounted for as an indispensable step for proper conservation and restoration of contemporary art, particularly happenings and performances, for their intrinsically ephemeral nature, and installation-based artworks for they consist of a very complex assemblage of parts, each of which requires documentation both separately and in their reciprocal interaction (Schölte, Wharton, 2011). In the effort of acknowledging the wide range of typologies of contemporary artworks, documentation in this field follows different approaches and adopt a variety of methods and techniques, often tailored on the specific object under observation. Some methodologies have been proposed in the past years and are still under improvement. This includes the production of models, artists' questionnaires and interviews, the development of checklists, templates and workflow procedures, instrumental analysis and, more recently, the adoption of newer imaging techniques like laser scanning, 3D video analysis and virtual reality panorama photography. A systematization of this multifaceted and fragmented panorama of documenting techniques is a current concern of conservation scholars and practitioners, who would benefit from clear guidelines assisting their hard work when dealing with contemporary artworks.

Furthermore, the totality of these methods unfortunately still falls short in documenting the intangible, ephemeral and perceptive features of complex immersive and movement-based installations/environments. In these cases, in fact, conservation priority should be the pursuit of the global effect of the artwork on the user's perception, if we want to ensure its survivance as a whole. A documentation campaign led by this specific aim could be a valuable asset for properly preserving, regenerating or re-installing such complex works of art.

An important effort to meet this challenge - along with the issue of technological obsolescence - emerged from the Guggenheim Museum's effort to preserve its world-renowned collection of conceptual, minimalist and video art (Depocas, Ippolito, 2003). It is represented by the Variable Media Network, a proposition of unconventional preservation strategies based on identifying ways that creative works might outlast their original medium. The tool developed within this paradigm is the Variable Media Questionnaire. Supported by an online platform, the questionnaire is an interactive form linked to an open database and is intended to spur questions that must be answered in order to capture creators' desires about how to translate their work into new mediums once the work's original one has expired. Questionnaire also recognizes environments, user interactions, motivating ideas, and external references as aspects to be surveyed and considered when preserving or recreating the piece [http://variablemedia.net].
The documentation of technology-based artworks - and of course their conservation - has specific complexities due to their operational requirements and mechanical or technological apparatus. In fact, as installations utilizing technology-based components - like the one proposed in this study - they exist as a whole only in their on-mode, matching physical and ephemeral presence, material and immaterial features (Bek, 2011). So, they need to be kept active. This particular condition entails that damaged or deteriorated parts may require a professional repair or even a replacement if we don't want to decommission them and consequently handle them as historical relics. If we accept this position, it's clear that we need to develop documentation strategies which don't limit to capture the state of the artwork yet are also suitable for guiding and managing properly the transformations that it will eventually undergo along its life. Documentation in this case plays a crucial role in enabling reparations, replacements, regeneration and re-installations of the artwork.

Also the diffusion of digital technologies offers a broad range of tools that can be adopted for documentation scopes alongside traditional and analogical ones. An interesting study dedicated to video documentation strategies, included in the EU Funded research project Inside Installations (2007), provides insights about the state of the art in this field. Nevertheless, there's still a knowledge gap about the available tools and technical methods specific to documentation of contemporary artworks. The technologies used are limited to descriptive databases and instruments for obtaining geometric measurements, together with the introduction of audio-video recordings. The tools and methods used for the digitisation of artworks are those in use for surveying built heritage, widely tested over the years. On the basis of geometric surveys - obtained with direct and indirect methods -, the conservative study usually defines the information to collect (Baratin, 2012; 2014; 2015).

The point of view changes when documentation is intended as a tool of transmission of the artistic meaning beyond the original medium (Muñoz Morcillo, Faion, 2016). In this case, the digitisation of an artwork is a way to preserve and make accessible its content over time. Digitisation can even be interpreted as an "upgrade of the medium of artworks to a contemporary standard, accepting any resulting changes in the look and feel of work". [...] "To emulate an artwork is not to store digital files on disk or physical artefacts in a warehouse, but to create a facsimile of them in a totally different medium" (Depocas, Ippolito, 2003).

Experimental researches about the virtualization of artworks' medium has been addressed mainly at New Media art, for which the replicability problem is a key topic (Wharton, 2015).

Summarizing, at present two different ways of designing and accomplishing an informal preservation model exist (Muños Viñas, 2005): a more technical one, useful to study any single component and re-install the artwork (Ferriani, Pugliese, 2017), and another more descriptive one that stems from the idea of virtualizing an artwork in order to create a kind of migrated sample useful for museum fruition (Empler, 2018) . In both cases the documenting process is of crucial importance for ensuring the proper transmission and interpretation of data, thus enabling a respectful, yet effective display of the artwork.

\section{A DIGITAL AND MULTIDISCIPLINARY DOCUMENTAL BOOKLET}

\subsection{The research goal}

The present research aimed at drafting a descriptive dossier of the various parts of the artwork, and developing an hands-on 
documental booklet that gathers together multiple sources of data. This goal entailed the exploration of the interdisciplinary role of conservators - who are in contact with different approaches and documentation practices - using an innovative and experimental documenting method.

Also, there are several technologies available for documenting objects belonging to cultural heritage, for collecting data and communicating them, from interactive databases to virtual and augmented reality. While accounting all the methods and techniques mentioned above, crucial could be the tuning of them towards the individuation of the actual subject of documentation and its end, be it preservation or fruition. Our research attempts to define a difference between documentation for conservation and documentation for fruition. In fact, the documentation priorities and outputs could change whether they address experts or a public of non-experts. This leads to the distinction of the methods of analysis, the gathering of data, and their rendering. So, if for the survey and study of the object/environment it is not enough to figure out a simple database with multiple entries, similarly a virtual or immersive methodology proves not to be the one and best choice for the representation of data.

We all know that museum professionals spend an increasing amount of time and resources in documenting technical details and conceptual underpinnings (Wharton, 2015), in the effort to provide knowledge to future needs. In this context, our research challenge and goal has been the achievement of a unique, clear and usable output obtained by weaving together a multi-input documentation. Using every necessary documentation tools, each one managed by a specific specialist, we achieved to design an experimental booklet for guiding conservators who deal with complex kinetic environments.

\subsection{Our case study}

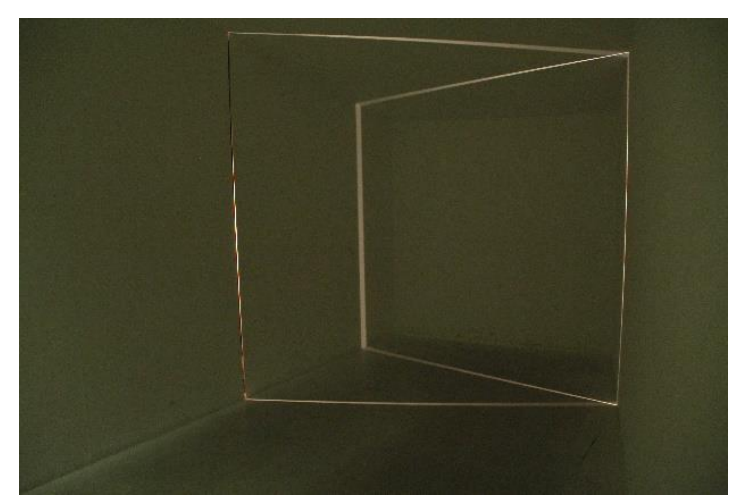

Fig. 1.Internal view of Ambiente - Strutturazione a parametri virtuali.

Ambiente - Strutturazione a parametri virtuali by Gabriele Devecchi is a kinetic-programmed environment created in 1969. Gabriele Devecchi (Milan, 1938-2011) has been a member of the italian Gruppo T, a founder group - together with Gruppo N - of Arte Programmata in 1962, mentored by Bruno Munari.

The environment has been repeatedly re-installed with slight ly different details across Italy and Europe through the following decades in occasion of temporary exhibitions, the last of which is currently on display at Museum der Moderner Kunst in Wien (1). The only permanent installation of Ambiente Strutturazione a parametri virtuali (Fig.1) dates back to 2010 and is displayed at Museo del Novecento in Milan. At that time the artist was still present and curated himself the

\footnotetext{
${ }^{1}$ Vertigo. Op Art and a history of deception 1520-1970, 25/06 26/10/2019, Museum Moderner Kunst, Wien
}

reconstruction, thus providing a benchmark for any other reproposition.

The version conserved in Milan consists of a trapezoidal blank space with white walls. Throughout two vertical slits in both of the sidewalls, two lines of light in movement draws continuously changing spatial references, putting in question the dimension and geometry of the space in the user's perception. The technological apparatus enabling this effect is hidden behind the sidewalls and is composed of two electromechanical devices with bulbs attached, assembled by the artist himself with industrial materials and handcrafted small pieces. The devices' function is to put in movement a total of 4 bulbs - two bulbs each - which rotate according to different programmed cycles thus tracing luminescent trajectories alternatively intertwining or departing one from the other. The user is invited to merge him/herself in the environment and experiment directly the puzzling effect of floating spatial references.

It's clear that in Ambiente - Strutturazione a parametri virtuali we can distinguish a tangible, material aspect - i.e. the complex of spatial, "architectural" features and technical apparatus - and a highly intangible one, i.e. the effect on users' embodied perception, which is given by the changing spatial parameters drawn by the movement of light. As said above, such an artwork exists as a whole only in its on-mode (Bek, 2010), i.e. when the tangible and intangible aspects synergistically express. Furthermore, as an immersive environment it can be considered complete only when people cross it. The artwork gets completed in the user's experience, which in turn requires an embodied engagement in space and the will of getting in the game.

For these reasons Ambiente - Strutturazione a parametri virtuali poses a wide spectrum of challenges to conservators, ranging from documentation issues to conservation strategies for ensuring the future proper fruition. In this essay, as already said, we focused on documentation-related problems, in the effort of gathering tangible and intangible data and giving them an accessible shape.

\section{THE METHODOLOGY: AN EXPERIMENTAL APPROACH}

The basic preservation strategy applied to our case study was structured in three phases, which involved the collaboration of various professional profiles according to the skills required for each specific job. Once the technical data had been collected, we focused on the study of the complexity of the whole environmental work. The sequential steps have been:

1. Collection of dimensional and geometric data. Goal: understanding the object;

2. Documenting movement. Goal: preserving the idea that resides in the effect of the object on user's perception;

3. Interrelate the data and assess their usability. Goal: describing the complexity of the work as a whole.

The third and last point contains a further documentation effort. In addition to storage and organization of information about the artwork's mechanical parts and aesthetics, the documentation structure also attempts to describe the relationships and interaction between these parts.

We have therefore defined 3 levels and/or 3 systems of relations:

3.1 Micro level/the lamp: it concerns the mechanical and functioning ratio of the lamp, how it moves and what is the role of each piece.

3.2 Macro level/ the environmental context: it explains the interaction between mechanical parts, their movement and their effects in the space; 
3.3 General level/fruition mode : the kind of relationship and interaction enacted by the user inside the artwork.

Establishing these three levels enabled us to put into relation the data analysed in the two previous phases towards the definition of a system. Below we will describe the three different steps of the documentation campaign.

\subsection{First step: the technical survey}

The first approach to the study and documentation of the artwork has been the metric and geometric survey of the lamps i.e. the mechanical and mobile parts - carried out by a professional restorer. The work has been organized as a traditional survey, using analogical instruments (millimeter meter, gauge, goniometer, etc..).

The subsequent rendering of measurements has been made by two-dimensional CAD systems, according to the rules of industrial design. Then, the overall dimensions have been reported on general diagrams and every part has been pinned on the side in a zoomed in scale (figg. 2,3). In fact, next to the technical drawing which includes orthogonal projections, an abacus of the single elements has been organised with indications about the material, functioning and specific dimensional references.

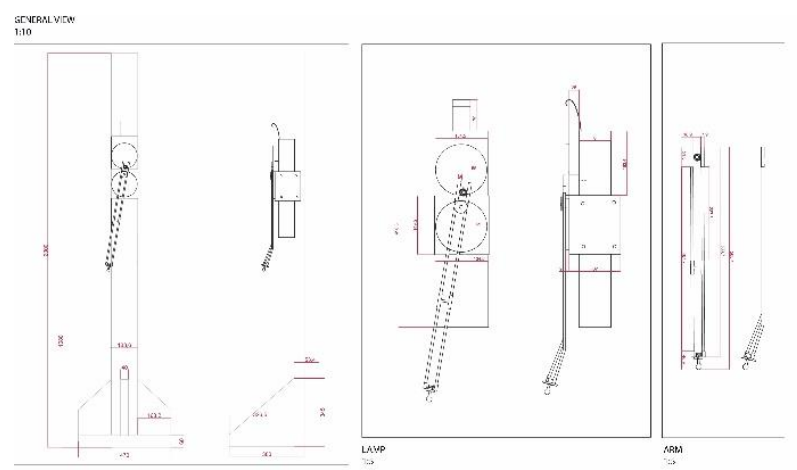

Fig.2.Components and overall dimension

The complete lamp module has been represented also thanks to the use of three-dimensional systems in an exploded view that indicates the assembly of the individual parts and the mechanical relationship between them.

In this phase, the survey also included a qualitative study of the object. The lamp was analysed distinguishing original and nonoriginal parts. This way, we proceeded to the mapping of maintenance interventions and manipulations underwent by the artwork over time. Maps have been made on a two-dimensional graphic basis, thanks to various layers that may be activated or not, according to the need.

Once the data collection phase was completed, from historyrelated to strictly technical ones, we moved on to the organization of data dissemination. We designed a clear hierarchical structure of the graphic database. The database is organized as follows: first the information necessary for understanding the operational aspects and in the background the technical data.

The result is a metric graphic model, implemented with various types of information, providing a graphic synthesis of the mechanical parts that enable the kinetism of the artwork.

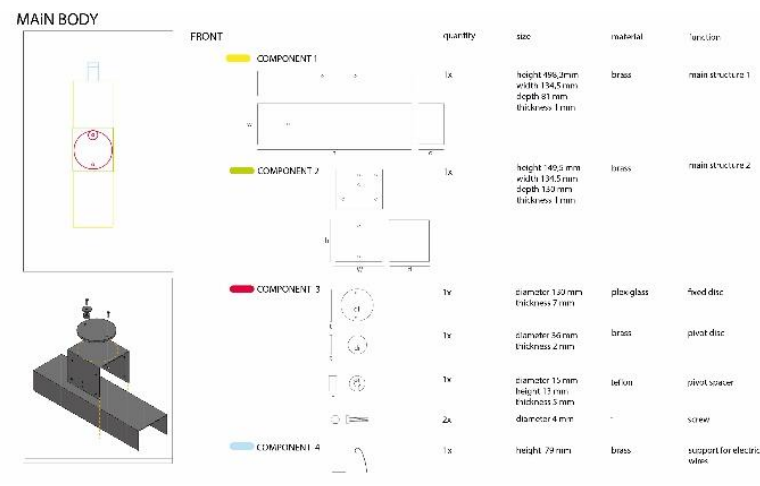

Fig.3.Abacus of the components

\subsection{Second step: the representation of the movement}

The second part of analysis required the close collaboration between the conservator who was implementing the documentation and a mechanical technician, who studied the functional mechanism of the lamps, drew the movement from a mathematical point of view and identified special features and defects of the object.

The movement of the lamps depends on two variables, which define the trajectory and speed of the light projection. Their functioning can be described as follows: a crankshaft mechanism translates the rotary movement of the upper disc and the oscillatory movement of the lamp, which unfolds in a trajectory that depends on the geometric parameters of whole system.

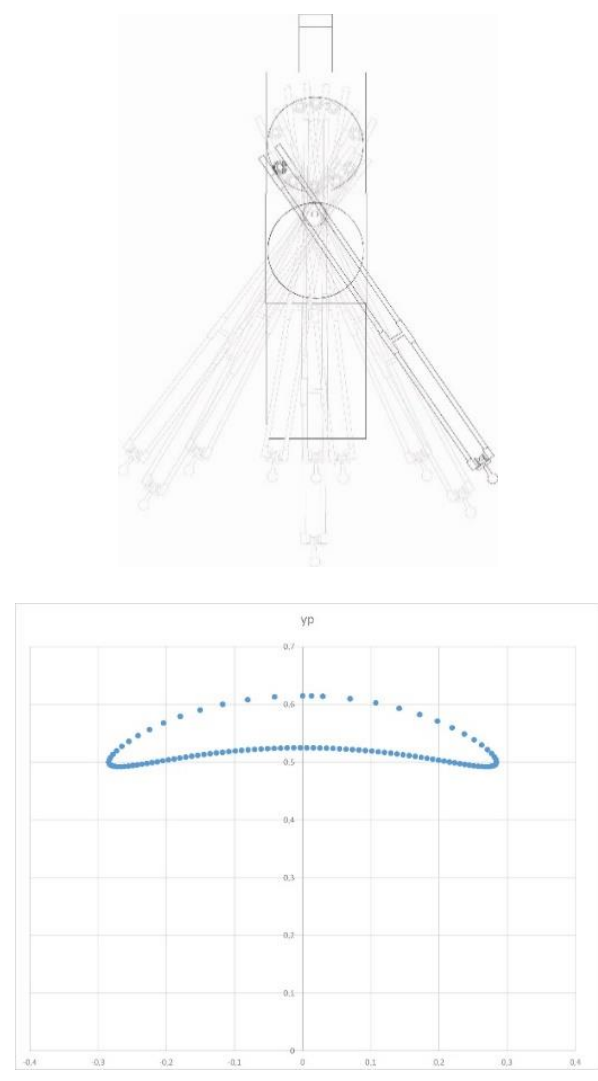

Fig.4.The image shows the qualitative trend of the lamp's speed by observing the distance between the various points of the trajectory graph. In addition, the trajectory reported corresponds to a series of crank rotations, which have a constant angular 
speed, of 0.06 radians (about 3.5 degrees). The most distant points (those in the upper part of the graph corresponding to the lamp at the bottom) correspond to a higher speed of the lamp, while those more closely packed together (high trajectory of the lamp) correspond to a slower movement.

Trajectory refers to the curve described by a point in motion relative to a given reference system. Therefore, to document this trajectory it is necessary to fix the geometry and dimensions of the entire system of the lamps. Starting from the surveys of lamp's elements, it is possible to define the path drawn by the bulb. In this specific case, the trajectory is shown on the graphic - overturned by 180 degrees for simplicity of construction (fig.4b). It consist in two "lunettes" whose lenght is about 30 $\mathrm{cm}$, by referring to the center of symmetry. This trajectory is drawn by the lamp with the same frequency as the crankshaft rotation set by the motor ( 5 revolutions per minute). The peculiar characteristic is the speed with which the lamp moves along the trajectory. This is not as constant as the speed of the motor, but it varies at all times according to the geometric parameters of the crank mechanism.

Moreover, thanks to a study of the projections, it is possible to identify the movement of the light across the room. However, this data are not fixed considering that the artwork can be reinstalled in spaces of different size - as happened in the past. By matching the two data sets - the graphical and mechanical one - we obtained the technical documentation necessary for the complete description of the work as a whole.

The representation of this analytical phase has been set according to the graphic-descriptive methods of the mechanical design. The result was a $2 \mathrm{D}$ drawing (fig.4a), realized with CAD systems, which associates physical-mathematical descriptions (formulas) with two-dimensional representations of objects.

Thanks to the collaboration between two professional expertise, it has been possible also to evaluate which are the descriptive data of the original functioning mechanism and which, instead, are related to degradation and therefore should be ignored in this documentary phase.

\subsection{Third step: the final model}

The documentation resulting from the first two steps provides many technical and "biographical" data about the artwork and allows its reconstruction on the basis of an authentical version, validated by the artist during the installation phase. It is a $2 \mathrm{D}$ and 3D graphic documentation and a descriptive mechanical documentation. The last step of this documentary path has been networking the data sets, inclusive of all the technical, comunicative and interactive information.

In first instance, this phase entailed the realisation of a descriptive video to stir in a static database. Neverthless, we have realized that when dealing with a complex artwork a video can be only a discrete part of an integrated documentation which instead should analyze the multiplicity of components of the whole environment. Admittedly, Gaby Wijers (2007) points out that video recordings should include multiple points of view, both subjective and objective. The resulting footage should be then edited, thus encompassing the inevitable influence of the editor's personal interpretation. That would transform documentation in a film.

The three levels of interaction (3.1 - 3.2 - 3.3) described above can be partially summarized with a virtual representation that shows the lamps' movement and the result of the light projection into the environment, possibly with one or more visitors passing by. Thus the random component involving the interaction user-environment would be represented only one way. Moreover, as a "flat" representation, a fundamental dimension is missing.

Again, contemporary ICTs allow the experimentation of new communication modes, useful to describe complex systems and to use them (Maniello, 2018). The methods of threedimensional modelling, of space virtualization and of augmented reality help to faithfully reproduce the environment without structuring a real informative system. Nevertheless, the artwork studied here no way can be reproduced with merely immersive systems. In fact, even if the third dimension is included, that indeed would provide a scientific restitution of data, though missing the immediate experience of the changing environment (no more real, but virtual). In this case it's not possible to render the artwork's configuration without the physical space and to translatate the physical space into a virtual space.

This solution would have dematerialised the original, creating a new (virtual) experience and not a simple digital copy. This type of digital representation falls short in documentating the level 3.3, i.e. the interaction of the whole system with the user. For this very reason, an immersive type of restitution has been excluded, which would have allowed the interaction with the virtual environment, yet without reporting the real data of the embodied physical experience.

Since we can hardly represent a random and subjective interaction, we should find a new representative method that doesn't limit to graphics and current digital instruments. We propose the use of ethnographic techniques and methodologies (Stigter, 2016; Pereira Marçal, 2017), which directly engages the user by asking him questions about his/her experience.Thus we may obtain a kind of emotional mapping, a perceptionrelated documentation with direct information about the use of the whole artwork.

Therefore, in this phase we worked with an online open database, which allowed us to structure a flexible format that could change, modulate and implement itself, according to the user's need. Within the database, questions have been proposed according to an approach similar to the Variable Media Questionnaire mentioned above (Depocas, Ippolito, 2003).

The user, after having experienced the artwork, can connect to the questionnaire by means of a digital tag. He/she can fill in the form with text, video, photos and audio. He also has a map of the environment, on which to report any information he/she considers worthy of attention.

This experimental phase still requires methodical tests (evaluated by visitors questionnaire given to a set number), Thus, we will obtain insights of various kind, from which starting to develop a trend map.

We strongly believe that a partecipatory documentation system would represent a vuable tool for acknowledging the intrinsic nature of the artwork.

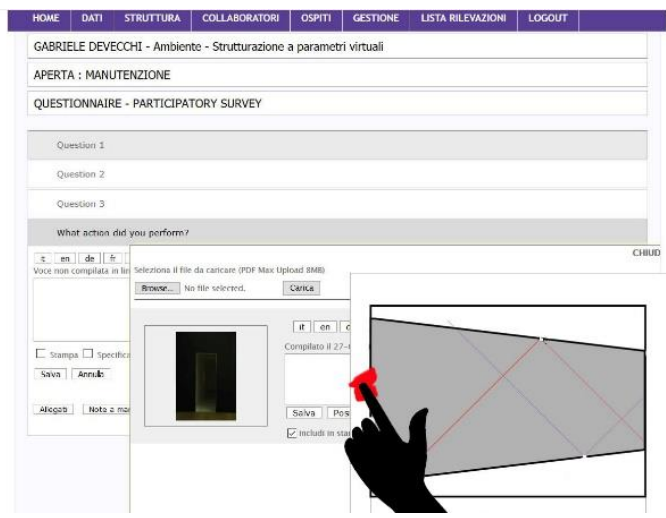

Fig.5. A way for visitors to use the database 


\section{CONCLUSIONS}

The study achieved an effective integrated documentation, comparing and using various technologies and methodologies. The result is a database, which can be tailored on specific needs, implemented over time and by several people working together. The database may be enhanced with graphic schemes, threedimensional representations and mechanical graphics.

The research aim has been to integrate several languages of documentation and representation. The available technologies have been evaluated and the actual usability has been assessed. Thanks to the collaboration of several professional figures, it's been possible to compare the methods of survey, choosing on each occasion the most suitable one.

As said above, the most innovati of technical data and for their description, considering that further researches should explore the integration and deepening also in the field of geomatics.

In the near future, we are planning to investigate further the role of ethnographic tecnhiques for interviews and survey of the users' experience inside the environment, in the effort of capturing the most intangible aspect of being immersed in Ambiente - Strutturazione a parametri virtuali.

As a general conclusion we may say that a research path has been drawn that will be developed in the future by keeping in consideration ever more participative approaches.

\section{ACKNOWLEDGEMENTS}

We would like to give a special thank to Archivio Gabriele Devecchi for the support provided in this research; to Museo del Novecento, Milano, for giving us access to the artwork in the survey phase; and to Mr. Paolo Crosa for the collaboration in the mathematical study of the lamps' movement.

\section{REFERENCES}

Baratin L., Antonelli E., Papi M., Salvarani M.P., Zuliani S., 2015. La collezione dei bozzetti lignei di Umberto Mastroianni: conoscenza e strategia per una conservazione e una valorizzazione funzionale. In: Dall'olio all'acrilico, dall'impressionismo all'arte contemporanea Studi, ricerche, indagini scientifiche ed interventi conservativi, Il prato publishing house srl, Saonara (PD), pp. 240-243.

Baratin L., 2014. Il Disegno E Le Opere D'arte: Problematiche Di Rappresentazione E Rilievo Nelle Scuole Di Restauro Dei Beni Culturali. In: Libro De Actas Del Congreso - Revisiones Del Futuro Previsiones Del Pasado V Congreso Internacional De Expresión Gráfica - Xi Congreso Nacional De Profesores De Expresión Gráfica. Rosario, Cues-Flashbay, pp. 50-57.

Baratin L., 2012. La documentazione prima del restauro:una problematica aperta. In: Arbace L., Baratin, L. (eds) Restauri d'Arte: opere dell'Abruzzo recuperate dopo il sisma. vol. 1, Gabbiano s.r.l., Ancona, pp. 148-152.

Bek, R., 2010. Between ephemeral and material-Documentation and preservation of technology-based works of art. In: Scholte T., Wharton (eds) Inside Installations. Theory and practice in the care of complex artworks, Amsterdam University Press, pp. 205-215.

Depocas, C., Ippolito, J. (eds), 2003. Permanence through change: the variable media approach, Solomon R. Guggenheim Museum, New York.
Empler, T., 2018. ICT per il Cultural Heritage. Dei Srl Tipografia del Genio Civile.

Ferriani, B., Pugliese, M., 2017. Lucio Fontana: Ambienti/Environments. Mousse Publishing.

Heydenreich, G., 2011. Documentation of change - Change of documentation. In: Scholte T., Wharton (eds) Inside Installations. Theory and practice in the care of complex artworks, Amsterdam University Press, pp. 155-172.

Maniello, D., 2018. Tecniche avanzate di video mapping Spazial Augmented Reality applicata al bene culturale. Le Penseur.

Muñoz Morcillo, J., Faion, F., 2016. E-Installation: Synesthetic Documentation of Media Art via Telepresence Technologies. In: Bostenaru Dan, M., Craciun, C. (eds.). Space and Time Visualisation. Springer, pp. 173-171.

Muños Viñas, S., 2005. Contemporary theory of conservation. Taylor \& Francis Ltd.

Pereira Marçal, H., 2017. Conservation in an era of participation, Journal of the Institute of Conservation, 40:2, pp. 97-104, DOI: 10.1080/19455224.2017.1319872.

Stigter, S., 2016. Autoethnography as a New Approach in Conservation, Studies in Conservation 61, sup2, pp. 227-32, DOI: $10.1080 / 00393630.2016 .1183104$.

Wharton, G., 2015. Public Access in the Age of Documented Art. Revista de História da Arte - Série W., Instituto de História da Arte, Lisbon, pp.173-184.

Wijers G., 2007. Video documentation of installations. Netherlands Institute for Cultural Heritage / ICN. 